\title{
Essential TRUThs AND Their TRUTH-GROUNDS
}

\author{
ROBERT MICHELS \\ eidos - the centre for metaphysics $\mathcal{E}$ University of Neuchâtel
}

\begin{abstract}
This paper motivates and defends a principle which captures a systematic connection between essence, truth, and grounding. It says that if a proposition expresses an essential truth, i.e., if it is true in virtue of the nature of some objects, then there are grounds for its truth which involve these objects. Together with the assumption that a fact can only be grounded in facts which are relevant to it, this principle is then applied in an argument against the monotonicity of the Essentialist notion 'true in virtue of the nature of'.
\end{abstract}

\section{Essential Truth and Ground}

There is a current trend in analytic philosophy of abandoning the austere view of metaphysics set out in Quine (1948) and of theorizing not only about which entities exist, but also about their essences and about structural relations of grounding which hold between facts containing them. A broadly Neo-Aristotelian approach to the notions of essence and ground has recently enjoyed much attention both in metaphysics, meta-metaphysics, and other philosophical sub-disciplines. ${ }^{1}$ An important open question about this approach, which has recently attracted considerable interest, is how these two notions relate. ${ }^{2}$ Inquiries into the relation between essence and ground are not only of intrinsic interest, but also promise new insights about each of the two notions, or so I will argue in this paper. The focus in this paper is on a particular principle, the Linking Principle, which

Contact: Robert Michels <mail@robert-michels.de>

1. For applications of the two notions outside of (meta-)metaphysics, see, e.g., Beddor 2015) (epistemology), Dasgupta (2014b), Garcia-Carpintero (2007), Kroedel and Schulz (2016), (philosophy of mind), Ellis (2001) (philosophy of science), Väyrynen (2013) (moral philosophy).

2. See, e.g., Carnino (2015), Correia (2013: in press), Correia and Skiles (in press), Dasgupta 2016), Fine 2012a: 2015), Rosen (2010), Trogdon (2013a). 
captures a systematic connection between essential truths and the grounds for their truth.

In Section 2. I will first introduce and motivate the principle and then defend it against objections. In Section 3. I will apply the principle to argue that the Essentialist notion 'true in virtue of the nature of' is not monotonic. Finally, in Section 4. I will respond to Tahko's (2013) conditional argument to the conclusion that truth-grounding is not grounding, which threatens to undermine the argument of the previous section, and more generally, the relevance of the Linking Principle to grounding. Section 5 briefly summarizes the main arguments of the paper.

\section{The Linking Principle}

\subsection{The Linking Principle Explained}

The dominant approach in the recent literature on essence is the one developed by Fine in a series of influential articles on essence and modality. ${ }^{3}$ One important feature of the Finean approach to essence is its focus on essential truths, truths which describe the essences of objects. Fine endorses the Aristotelian idea that there are 'real definitions', the idea that 'just as we may define a word, or say what it means, so we may define an object, or say what it is' (1994 2). In Fine's framework, claims about essence are canonically stated using the primitive Essentialist notion 'true in virtue of the nature of'. The claim that it is essential to Xanthippe that she is human translates to the claim that it is true in virtue of the nature of Xanthippe that she is human and her essence or real definition is identified with the set of propositions which are true in virtue of her nature (see Fine 1995c 55). Fine's notion also allows one to formulate claims about the essences of pluralities of objects, such as 'That Xanthippe is distinct from the Notre-Dame Cathedral is true in virtue of the nature of Xanthippe and the NotreDame Cathedral.' I will rely on plural variables $(x x, y y)$ and plural quantifiers when discussing such claims and will, for the sake of simplicity, treat single objects as limiting cases of pluralities. ${ }^{4}$

Fine's focus on essential truth marks an important contrast to the more common approach to essence which mainly focuses on essential properties. ${ }^{5}$ This

3. See Fine (1994: 1995c: 1995a: 2000) and Correia 2006: 2012) for further developments of the approach. See also Lowe (2008). and logic.

4. See Linnebo (2014) and Oliver and Smiley (2013) for introductions to plural quantification

5. See, e.g., Bennett (1969), Mackie (1994), Gorman (2005), Robertson and Atkins (2013), or Roca-Royes (2011). Note that in Fine's works, this seems to be first and foremost a contrast in how claims about essence are expressed. Indeed, the discussion of essence in Fine (1994) and Fine (1995C) is still partly framed in terms of essential properties. The shift of focus is most 
can be best brought out by considering an example. Suppose that a particular composite objects $c$ essentially has a distinct object $d$ as a proper part. ${ }^{6}$ Given this supposition, one may claim that it is essential to $c$ that $d$ exists. According to the more common approach to essence, this is reflected in $c^{\prime}$ s essentially having the property of being such that $d$ exists, on the Finean approach, this is reflected in the proposition $\langle d$ exists $\rangle$ 's being true in virtue of the nature of $c .^{7}$

Whether objects can have relational properties of this sort is controversial. A property of this kind does not carry it on its sleeve that it is a property which can be had by an object like $c$, in contrast to, for example, the relational property of having $d$ as a proper part. Unlike the former property, the latter has, metaphorically speaking, a slot for objects like $c .^{8}$ A related reason for suspicion regarding similar relational properties is that, given that $d$ exists, the property of being such that $d$ exists fails to genuinely contribute to characterizing the objects which have it, since it is trivially had by any object whatsoever which coexists with $d .{ }^{9}$

In contrast, it is uncontroversial that propositions which do not involve certain objects can describe them. The relation between a proposition and the objects it describes is less intimate than the relation between a property and the objects which have it. The general point, put in slogan-form, is that by shifting the focus from essential properties to essentially true propositions, Fine detaches the object from its essence.

Fine's shift of focus to essentially true propositions raises new questions about the relation between essence and ground and in particular about the grounds of the truth of essentially true propositions. Two such questions are first, whether there is a ground for the truth of each essential truth and, second, if so, what that ground is. The principle which I will motivate and defend in this section gives a positive answer to the first question and a partial answer to the second.

Before I introduce the principle, a few introductory remarks on the notion of ground are in order. It plays a central role in the Neo-Aristotelian approach towards a structured ontology. ${ }^{10}$ Examples of grounding-claims include 'The

noticeable in his formal work on the logic of essence, e.g., in Fine (1995a).

6. See, e.g., Chisholm (1973) for a defence of a modal interpretation of this claim.

7. I use angular brackets ' $\zeta\rangle^{\prime}$ to form names for propositions throughout this paper.

8. A similar worry is also raised in Oderberg (2011. 100, Footnote 46) in the context of a discussion of the restriction of the notion of consequential essence introduced in Fine $1995 \mathrm{C}$ $56-58)$.

9. Considerations of this sort are relevant in the context of the metaphysical discussion about the distinction between an abundant and sparse conception of properties. For discussions of the relevance of this distinction to the Esssentialist theory of modality, see Cowling (2013), Skiles 2015), Wildman (2013).

10. For overviews of the literature and introductions to ground, some opinionated, see Bliss and Trogdon (2016), Clark and Liggins (2012), Correia and Schnieder (2012a), Raven (2015), Trogdon (2013b). Articles introducing and defending the notion include Audi (2012b), Fine 
existence of the whole is grounded in the existence of its parts' or 'Mental facts are grounded in neuro-physiological facts. ${ }^{11}$ There are controversies about the grammar and the interpretation of the notion of ground or grounding. ${ }^{12}$ Following Rosen (2010), I will here assume that grounding claims involve a relational predicate and that grounding is a many-one-relation which holds between a fact or a plurality of facts, the ground or grounds, and another fact, the grounded fact. I will also follow Rosen and many others in assuming that grounding claims capture (parts of) metaphysical explanations. More could of course be said about essence and grounding, but these brief remarks and pointers should allow one to get enough of an idea about these notions to understand the principle which I am mainly interested in here:

Linking Principle For all pluralities of objects $x x$, if a proposition $p$ is true in virtue of the nature of $x x$, then there are some facts involving all of the $x x$ which together fully ground the fact that $p$ is true. ${ }^{13}$

In other words, the principle says that if a proposition captures part of the essence of some objects, then its truth is grounded in some facts involving those objects. ${ }^{14}$ To give an example, if Socrates is essentially human, the Linking Principle tells us that there exist some facts which fully ground the truth of 〈Socrates is human〉 and that at least one of these facts contains Socrates. Note that the principle places no restrictions on how the objects quantified over in its antecedent distribute over the facts quantified over in its consequent. They may, for example, be evenly distributed, so that each object is involved in its own fact, or they may all be involved in just one of the grounding fact, or in all of them, etc.

2012a), Rosen (2010), Schaffer (2009). For a discussion of the relation between grounding and existential dependence, see Schnieder (in press). Critical voices include Della Rocca (2014), Wilson (2014).

11. See Correia and Schnieder 2012a: 1) for further examples.

12. See Correia and Schnieder (2012a: Section 3.1).

13. For the sake of simplicity, I work with a simple property/relation-based view of facts throughout this paper. Concerning the individuation of facts, the conception more or less matches Audi's worldly conception of facts (see Audi 2012b 696-697) in that facts are individuated by their non-factual components, i.e., the properties, relations and objects they contain, and their arrangement and in that differences in how these components can be described do not matter for fact-individuation. Note that the view does not incorporate Audi's controversial 'thick' conception of worldly facts, according to which there are, e.g., no existence or identity facts. (See Audi $2012 b$ 700 \& 705, Footnote 34.)

14. The following is a more formal re-statement of the principle: $\forall x x\left(\square_{x x} p \rightarrow \exists \mathrm{ff}(\mathrm{ff}\right.$ $[T p] \wedge \forall y(y \leq x x \rightarrow \exists \mathrm{f}(\mathrm{f} \sqsubseteq \mathrm{ff} \wedge y$ If $))))$. ' $\square_{x x} p^{\prime}$ stands for ' $p$ is true in virtue of the nature of $x x^{\prime}$, the statement involves singular and plural quantification over fact-variables $\mathrm{f}$, $\mathrm{ff}$, ' $\mathrm{ff} \longrightarrow[T p]^{\prime}$ stands for 'ff fully ground the fact that $p$ is true'. Square brackets ' [ ]' are used to form names of particular facts, ' $y \leq x x^{\prime}$ stands for 'object $y$ is among the objects $x x^{\prime}$, ' $\mathrm{f} \sqsubseteq \mathrm{ff}$ for 'the fact $\mathrm{f}$ is among the facts $\mathrm{ff}^{\prime}$, and ' $y \mathrm{If}$ ' for 'object $y$ is involved in fact $\mathrm{f}^{\prime}$. 
The Linking Principle makes a generic claim about the truth-grounds of essentially true propositions, which means that different pluralities of facts may satisfy its consequent for each such proposition. Which facts these are may depend on the relevant claim about essence. To give an example, assume that $\langle$ Xanthippe is distinct from the Notre-Dame Cathedral $\rangle$ is true in virtue of the nature of Xanthippe and the cathedral taken together. If [ $\langle$ Xanthippe is distinct from the Notre-Dame Cathedral $\rangle$ is true] is grounded in [Xanthippe is human] and [The Notre-Dame cathedral is not human], then the Linking Principle is satisfied. ${ }^{15}$

It is important to distinguish the principle from a distinct, but similar principle, which says that if a proposition captures part of the essence of some objects, then the fact described by the proposition is grounded in some facts involving these objects. Facts of the form $[\langle p\rangle$ is true] are distinct from those of the form $[p]$ and these two sorts of facts do in general not have the same exact grounds, since, for example, $[p]$ grounds $[\langle p\rangle$ is true], but not vice-versa.

There is also an important remark on the notion of 'true in virtue of the nature of' as used in the Linking Principle, which should be made. In Fine (1995C), Fine distinguishes the constitutive essence of objects, which is formed by those propositions which describe what is 'directly definitive' (1995C 57) of them from their consequential essence, which contains propositions which logically follow from their constitutive essence. The constitutive notion is arguably the notion which is at work in Fine's (1994) important objections to the modal definition of essentiality (see Michels in press). In the Linking Principle, 'true in virtue of the nature of' expresses constitutive essentiality. ${ }^{16}$ If it expressed an unrestricted notion of consequential essentiality instead, or in addition to constitutive essentiality, the principle would, for example, have the consequence that the truth of $\langle$ The Eiffel Tower is a building or not a building $>$ is grounded in some facts about Socrates,

15. Note that in general, a fact may have multiple, distinct full grounds. In this case [Xanthippe is not a building] and [Notre-Dame is a building] may together provide an alternative full ground.

16. I hence agree with Correia (in press) that it is admissible for Essentialists to work with the constitutive notion. Fine in contrast proposes 'to work as far as possible with the consequentialist notion' (1995C 58) and, to avoid overgeneration-problems, restricts this notion. In Fine 1995C 58-60) the consequentially essential properties of objects are restricted to those involving objects which cannot be generalized out of the consequential essence in a manner described there. Furthermore, the consequential notion used in Fine's logic of essence is also subject to a restriction based on the objects on which the objects in virtue of which a proposition is true depend. See Fine (1995a 253, Theorem 4) and the semantics developed in Fine (2000). For discussions of Fine's 'generalizing-out'-restriction which question whether it suffices to give us an analogue of the constitutive notion, see Koslicki (2012 Section 7.3), Oderberg (2011. 100), Correia (in press Sections 5 \& 7). I do not wish to enter this discussion here, but would like to point out that if there is a restriction of the consequential notion which adequately captures the constitutive notion, I see no reason why one should not be able to rely on this restricted consequential notion in the Linking Principle. 
thereby violating a commonly accepted relevance-requirement for grounds. ${ }^{17}$ This qualification should be kept in mind, since I will later use the principle to argue against the monotonicity of the constitutive notion of 'true in virtue of the nature of', a property which the consequential notion uncontroversially has.

Before I go on to discuss the motivation for the principle, it should furthermore be pointed out that if truthmakers are truth-grounds, the Linking Principle corresponds to a restricted version of Truthmaker Maximalism, the claim that every truth has a truthmaker. ${ }^{18}$ This raises the general question of whether we may be able to simply identify truthmaking and truth-grounding. This attractive idea is threatened by an argument presented in Tahko (2013), which suggests that truthmaking cannot be explicated in terms of truth-grounding. I will respond to Tahko's argument in Section 4, not only to show that identifying the two notions is still an option, but also to address objections to the main argument of Section 3 and to the relevance of the Linking Principle to grounding which derive from Tahko's argument. Before that, the relation between truthmaking and truth-grounding will already play an important role in the next subsection.

\subsection{Motivating the Linking Principle}

\subsubsection{The Essential Truthmaking-View}

In this subsection, I will present two motivating arguments for the Linking Principle. The first argument is based on the idea that 'true in virtue of the nature of' is a special case of truthmaking, namely essential truthmaking. ${ }^{19}$ This idea meshes well with the established view that truthmakers are 'entities in virtue of which sentences and/or propositions are true' (Mulligan, Simons, \& Smith 1984: 287). ${ }^{20}$ Based on a realist reading of this quote, we can take truthmaking to be a relation which relates entities and propositions. Fine's notion 'true in virtue of the nature of' can in a similar spirit be taken to stand for a relation which takes one or more objects and a proposition as its relata.

According to this realist view of the two relations, they are both crosscategorial, in the sense that they relate entities existing in the world, which may belong to any category, including that of propositions, to propositions. Since

17. This follows since, assuming a classical notion of logical consequence, the proposition 〈The Eiffel Tower is a building or not a building follows from any proposition and for that reason belongs to the unrestricted consequential essence of any objects whatsoever.

18. See MacBride 2013 Section 2.1) and Rodriguez-Pereyra (2006b) for recent overviews of the discussion about Truthmaker Maximalism.

19. The following presentation of this view owes a lot to comments of an anonymous referee for this journal, who in particular suggested that the view may be usefully spelled out in terms of determinables/determinates and the label 'true in virtue of the existence of' for non-essential truthmaking.

20. See also Rodriguez-Pereyra 2002 34) and Rodriguez-Pereyra 2006b 187). 
we can assume that truthmakers can also be objects, instead of, for example, only facts (see Armstrong 2004: 6), it appears that we can claim that to be true in virtue of the nature of some objects is a special case of truthmaking. ${ }^{21}$ To be more specific, the claim is that the relation corresponding to Fine's notion is a (strict) sub-relation of the truthmaking-relation, in the sense that any instance of the former is also an instance of the latter and that the converse does not hold. ${ }^{22}$ This is the sense in which the resulting view, which I will call the essential truthmaking view, treats 'true in virtue of the nature of' as a special case of truthmaking.

The essential truthmaking view is supposed to be conservative with respect to the Finean notion 'true in virtue of the nature of'. Since this notion allows for essential truths about pluralities of objects, as pointed out in Section 2.1. the view has to make room for instances of essential truthmaking in which several objects contribute to making a proposition true. There are two way of doing this, one which is more conservative, the other more revisionary regarding the notion of truthmaking. The conservative way maintains that there is only a single truthmaker which involves all the relevant objects. Candidate entities for playing this role might, for example, be facts, or perhaps mereological sums of these objects. Whatever choice one makes, the truthmaking-relation will, again metaphorically speaking in terms of slots, remain singular in its truthmaker-slot. The revisionary way, in contrast, allows these objects together to plurally stand in the essential truthmaking relation to the relevant proposition, making the relation multigrade in its first argument-place. Since the essential truthmaking view takes the essential truthmaking-relation to be a (strict) subrelation of the general truthmaking relation, this entails that truthmaking in general has to be plural too.

The choice between the two options involves a trade-off between conservativeness regarding the notion of truthmaking (hold on to the standard view that truthmaking is singular) and the Finean notion 'true in virtue of the nature of' (hold on to the standard view that this notion is plural). In introducing the view, I have focused on the latter option, but the first also seems viable. I will not further discuss this issue here, since both options give us versions of the view which support the Linking Principle.

The central claim of the view is formulated in terms of (classes of) instances of the two relations, but one may of course ask what the view tells us about the relations themselves. For the purposes of this paper, I do not want to commit

21. If one admits only facts as truthmakers, one would instead have to claim that the 'true in virtue of the nature of'-relation takes a fact and a proposition as its relata. This alternative view is obviously more revisionary, regarding the treatment of Fine's notion.

22. More precisely: If $T$ is the class of all instances of the truthmaking-relation and $E$ the class of all instances of the relation corresponding to 'true in virtue of the nature of', where the instances of these relations are ordered sets of the entities which stand in the respective relation, then $E \subsetneq T$, i.e., $E$ is a proper subclass of $T$. 
myself to any particular metaphysical view, but I still want to mention one which appears plausible. According to it, truthmaking is a determinable and essential truthmaking one of its determinates. ${ }^{23}$ The more familiar relation which holds between objects and propositions irrespective of their essences is another determinate of truthmaking, for which one might introduce the notion 'true in virtue of the existence of'. There is more to say about this proposal, but this brief remark should suffice to illustrate that the essential truthmaking-view can coherently be fleshed out in metaphysically more substantial terms.

It should also be mentioned that the purpose of the essential truthmaking view is not to offer a substantive analysis or a reductive definition of what it is to be an essential truth. In this respect, the view follows Fine's suggestion to treat the notion of essence as primitive: What distinguishes instances of essential truthmaking from other instances of truthmaking is simply that the related entities stand in a special sort of truthmaking relation, a relation which only holds between an object (or several objects) and a proposition which captures part of the essence, or equivalently, of the real definition of this object (or these objects).

The essential truthmaking view entails that essentially true proposition trivially have a truthmaker involving all objects whose essence they describe. ${ }^{24}$ In order for this to motivate the Linking Principle, we still need to establish that for an essentially true proposition to have a truthmaker implies for it to also have a truth-ground, that is, a fact which grounds its truth. In most cases, such facts are easy to come by. Think, for example, of 〈Socrates is human〉 and the corresponding fact [Socrates is human]. A problem might arise if there are propositions which are true in virtue of the nature of some objects, but fail to involve these objects. The example of $\langle d$ exists $\rangle$ 's being true in virtue of the nature of $c$ which has $d$ as a proper part from Section 2.1 fits this mould. Like other purported essential truths of this sort, the example involves substantial metaphysical assumptions which one may reject. Be that as it may, the second motivating argument offers a way to close this potential gap in the first argument.

\subsubsection{Facts About Essences as Truth-Grounds}

There is another, perhaps more general argument for the Linking Principle on which one might either rely on its own or in conjunction with the first argument. ${ }^{25}$ This second argument for the Linking Principle is based on the assumption that true claims about essences always correspond to facts about essences. Such facts must involve the objects in virtue of whose nature the relevant proposition is true,

23. See Wilson (2017) for an introduction to this distinction.

24. If the previous suggestion to treat essential and the more familiar existential truthmaking as two determinate of the determinable truthmaking is accepted, truthmaking has to be a determinable which allows for the co-instantiation of two of its determinates.

25. Thanks to Mike Raven for suggesting this argument.

Ergo $\cdot$ vol. 5, no. $30 \cdot 2018$ 
even if these objects are not involved in the proposition. The assumption tells us that if it is, for example, true in virtue of the nature of Socrates that he is human, then there is a fact that the proposition saying this is true in virtue of the nature of Socrates. Only one further step is needed to complete the argument for the truth of the Linking Principle: It has to be established that this very fact fully grounds the truth of the proposition involved in it. To be sure, there is no doubt that it entails the latter fact, ${ }^{26}$ after all, a proposition cannot be essentially true and still fail to be true. But one might still doubt that this entailment indicates an instance of grounding.

Are there cases in which an entailment indicates an instance of grounding? I claim that there are, at least if the entailment is an entailment to the truth of a proposition. Not any such entailment will do however. First, the particular entailment must of course not conflict with the formal properties of grounding (see, e.g., Fine 2012a; 2012b). To give an example in which such a conflict occurs: [ $\langle p\rangle$ is true] trivially entails itself, but this fact cannot ground itself, since grounding is irreflexive.

A second condition is that the entailing facts have to explain the entailed fact. In the case under consideration, that is indeed the case: If I ask you to explain to me why it is true that Socrates is human and you respond that that is true because being human is one of Socrates's essential properties, then you have given me a sensible explanation (see, e.g., Dasgupta 2016). Of course, it would have been just as reasonable to respond that this is true because being human is one of Socrates's properties or just that Socrates is human, but this is not a problem. A fact may very well have several different, but equally admissible explanations.

The consideration of the previous paragraphs also illustrates why the second argument can be used to close the potential gap left by the first argument, if there indeed is such a gap. The gap concerns cases in which a proposition is true in virtue of the nature of objects other than those involved in it. The following example illustrates why the second argument supports the truth of the Linking Principle in such cases: If it is true in virtue of the nature of composite object $c$ that its proper part $d$ exists, then the fact that this proposition is true in virtue of the nature of $c$ gives us a full ground for the truth of the proposition $\langle d$ exists $\rangle$.

There is a sort of essential truth which, the second argument notwithstanding, still might pose a threat to the Linking Principle. It is sometimes claimed that a proposition can be true in virtue of the nature of no objects at all. In such a case, it seems that there simply are no objects which could be involved in the proposition's truth-grounds. To complement the two arguments for the truth of the Linking Principle, I will defend the Linking Principle against this and two

26. I here take the liberty of loosely talking of entailment between facts whenever there is an entailment among the corresponding propositions. 
further objections in the following section. ${ }^{27}$

Before I do that, I will discuss an aspect of the Linking Principle which will be of great importance later on in the paper. The Linking Principle tells us that there are facts which together give us a full ground for the truth of any proposition which is true in virtue of the nature of some objects and that these facts involve all of these objects. One might argue that the two motivating arguments of this section equally well support a logically weaker version of the Linking Principle, according to which only some of these objects need to be involved in the grounding facts. This weaker version of the principle is satisfied in all cases in which the stronger version is satisfied, so one might argue that this, rather than the stronger version of the principle, should be accepted.

Why should one worry about these two different versions of the principle? One reason is that the choice makes a difference regarding the argument against the monotonicity of the Finean notion of 'true in virtue of the nature of' which will be presented in Section 3. This argument requires the stronger version of the Linking Principle. The weaker version does not suffice, so the argument could be resisted by arguing that the weaker, rather than the stronger principle should be adopted. ${ }^{28}$

My response to this objection is that Essentialists should adopt the stronger version because it is preferable to the weaker version for a metaphysical reason. The reason is that the weaker version is clearly at odds with the metaphysics of some situations in which the principles are satisfied. This can be illustrated in two ways, neither of which involves considerations about monotonicity which would beg the question against the objector.

First, recall that according to the second motivating argument, the Linking Principle is always satisfied, because the truth of the proposition stating an essential truth is always fully grounded in the fact that the proposition is true in virtue of the nature of the relevant objects. Each such grounding fact trivially involves all the objects whose essence is (partly) captured by the proposition, not only some of them. The weaker version however leaves it open whether all objects are present in the full truth-grounds, contrary to what the metaphysics tells us.

27. The literature on truthmaker maximalism suggests further objections to the Linking Principle, but responses are likewise readily available to defenders of the principle. E.g., one might argue that, assuming that necessary truths lack truthmakers (see, e.g., Mellor 2003 213; Cameron 2008; and Contessa 2010 for recent discussions), essential truths do too, or that Milne's (2005) self-referential truthmakerless sentence expresses an essential truth whose truth goes ungrounded, or that the same holds for true negative existential propositions which express essential truths about some objects (see, e.g., Mulligan et al.|1984 313). To very briefly respond: Armstrong (2004 Chapters 5 \& 6) offers a response to the third objection, Rodriguez-Pereyra (2006a), Barrio and Rodriguez-Pereyra (2015) to the second. The first objection will not phase Essentialists, since the Essentialist reduction of necessity to essence commits them to the existence of grounds for necessary truths (see, e.g., Fine 1994, Correia 2012).

28. I am grateful to an anonymous referee for raising this objection.

Ergo • vol. 5, no. $30 \cdot 2018$ 
The second way in which the weaker version fails to match the metaphysics concerns cases in which a proposition (partially) captures the essence of a plurality of several objects, but not the essence of any of the single objects involved. A standard example of an irreducibly plural essential truth of this kind is the proposition 〈Socrates is distinct from the Eiffel Tower $\rangle$. Since Socrates and the Eiffel Tower are not essentially connected to each other, the proposition can describe neither the individual essence of the one, nor that of the other of the two objects (see Fine 1994: 5). This situation is mirrored by the truth-grounds of the proposition: Neither facts involving only Socrates, nor facts involving only the Eiffel Tower, can on their own fully ground the truth of the proposition which says that the two objects are distinct. The weaker version of the Linking Principle again misleadingly leaves open the possibility that a fact involving only one of the relevant objects may play the role of a truth-ground. It is again the stronger version of the principle which gets the metaphysics of these situations right. Since the Linking Principle is supposed to get the metaphysics right, friends of grounding and essence should accept the stronger, rather than the weaker version of the principle.

\subsection{Objections to the Linking Principle}

\subsubsection{Vacuous Essential Truths}

The logic of essence developed in Fine (1995a) admits propositions which are true in virtue of the nature of no objects at all, or, as Fine puts it, which are true 'regardless of the nature of any objects' (1995a: 246). This feature of the logic suggests the following objection: Since in the vacuous case there are no objects in virtue of whose nature the relevant proposition is true, there are also no facts involving these objects which ground the truth of the essentially true proposition. Therefore, the Linking Principle does not hold. ${ }^{29}$

There are at least two ways to respond to this objection. First, one may point out that the fact that the logic of essence allows for vacuous essential truths does not mean that they are also in the relevant range of applications of the Linking Principle. This is so because the notion 'true in virtue of the nature of' captured by Fine's $\square_{F}$-operator of the logic of essence expresses a different notion of essence than the notion which occurs in the Linking Principle. The logical operator captures the notion of consequential essence which includes logical consequences of essential truths. However, as I have pointed out previously, the Essentialist notion involved in the Linking Principle has to be read as expressing constitutive

29. Note that this objection is more plausible with respect to a more formal statement of the Linking Principle if one follows Fine (1995a) in using rigid predicates instead of plural variables in the index of his essentialist operator. Given this approach, vacuous essential truths can be taken to involve an empty rigid predicate. 
essence instead. It might make sense to allow vacuous consequentially essential truths, but it hardly makes sense to allow them in case of the constitutive notion. The constitutive notion captures the intuitive notion of essence according to which an essentially true proposition always captures an aspect of the essence of the objects it expresses an essential truth about. In the vacuous case however, there simply are no such objects and therefore, there is also no essence which could be captured by the essentially true proposition. With a nod to Carnap's (1932) famous warning against objectifying talk about nothing, the first response hence is that the idea of vacuous constitutively essential truth does not make sense.

The first response has my sympathies, but there is a second, more ecumenical response. One might argue that empty constitutive essential truths pose no problem for the Linking Principle, because for every proposition true in virtue of no objects at all, there are some objectually empty facts which ground its truth. This is consistent with the only available indication of how to understand the vacuous case, which is proposed (but not endorsed) in Fine (1995a: 250). According to this proposal, if one assumes that the domain of entities with essences only includes the objects, not the concepts, one can identify the objectually empty essential truths with the conceptual necessities. If one furthermore assumes that conceptual necessities have no objectual content (e.g., because all conceptual necessities have the form of universally quantified sentences involving no terms referring to individual objects) and in addition that they correspond to objectually empty facts, one has everything one needs to ascertain that the Linking Principle is satisfied in cases of vacuous essential truth. To illustrate this using an example: Assume that $\langle$ All bachelors are unmarried $\rangle$ expresses a constitutively essential truth about no objects and therefore a conceptual truth. According to the second response, the truth of this proposition is fully grounded in the fact [All bachelors are unmarried], a fact which contains no objects.

\subsubsection{Essential Truths as Autonomous}

Dasgupta has recently argued that essential truths, or in his terms, essentialist truths, are autonomous, that is, 'not apt for being grounded in the first place, in roughly the same way that arithmetic facts are not apt for causal explanation and that definitions are not apt for proof' (2016: 386). One might perhaps think that his view undermines the Linking Principle, but a closer look at what Dasgupta means by 'essentialist truth' shows that this is not the case.

An essentialist truth, or rather an essentialist fact in Dasgupta's sense (he seems to use the two terms interchangeably) is 'not the fact that $\phi$ but rather the fact that it is essential to $x x$ that $\phi^{\prime}$ (Dasgupta 2016. 387). This means that Dasgupta's autonomy claim is a claim about the whole antecedent of instances of the Linking Principle (i.e., about 'a proposition $\langle p\rangle$ is true in virtue of the nature of a plurality of objects $\left.x x^{\prime}\right)$. The claim in the consequent is in contrast 
only about the truth of the proposition involved in this claim. To illustrate this using one of Dasgupta's examples, suppose that it were essential to knowledge for it to be justified, true belief. The corresponding fact, the fact that it is essential to knowledge that it is justified, true belief, would then not be apt for being grounded. But the corresponding instance of the Linking Principle would not require there to be a ground for this fact. Rather, it would only require there to be some facts which ground the truth of the proposition that knowledge is justified, true belief.

The general point is that the consequent of the Linking Principle is only concerned with the grounds for the truth of the proposition describing the 'that $\phi^{\prime}$-part of an essentialist fact in Dasgupta's sense. The principle is perfectly compatible with the latter fact's not being apt for being grounded. If one takes into account what Dasgupta means by essential truth or fact, it is plain to see that there is no direct conflict between the Linking Principle and his autonomy claim.

This still leaves room for a more subtle objection, according to which the principle and the autonomy claim still conflict if one admits iterated essentialityclaims, that is, claims which say that it is true in virtue of the nature of some objects that it is true in virtue of the nature of some objects that .... Given a true proposition expressing a claim of this sort, the Linking Principle implies that there is a full ground for the fact that the embedded essence claim is true. Now if we assume that a ground of a truth-fact is always also a ground of the fact corresponding to the proposition involved in the truth-fact, which in this case would be an essentialist fact in Dasgupta's sense, autonomy would be violated.

To avoid this more subtle objection, a defender of the Linking Principle could either outright deny the sensibility of iterated constitutional essence-claims (see Dasgupta 2014b: Section xi, and Glazier 2016: Section 4 for arguments which point in this direction), or deny that the ground of a truth-fact is always also a ground of the fact corresponding to the true propositions involved in the truth-fact. If one opts for the latter response, one may for example argue that if a corresponding iterated essence claim is true, [ /It is true in virtue of the nature of Socrates that he is human〉 is true ]] is grounded in [ It is true in virtue of the nature of Socrates that he is human ], but that the latter fact remains ungrounded. I take both to be viable responses, so the more subtle version of the conflict can also be avoided. ${ }^{30}$

\subsubsection{Essential Explanation as Sui Generis}

Glazier (2016: Section 3) argues against the claim that if it is a fact that it is essential to $x x$ that $p$, then that fact about the essence of $x x$ grounds the fact that $p$. Like Dasgupta, Glazier assumes that grounding is linked to metaphysical

30. Thanks to Fabrice Correia and Claudio Calosi for raising this version of the objection and for discussion. 
explanation. His general idea however is that facts about essences explain other facts in a sui generis way, giving us a form of metaphysical explanation which is distinct from grounding.

Glazier relies on an example to support the negative part of this claim. Suppose that it is essential to a boolean variable in a computer program that it either has value o or value 1 . This fact about the essence of the variable explains the disjunctive fact that the variable either has value o or value 1. Glazier argues that this explanation cannot be an explanation of the sort which indicates grounding, for the following reason. By a general principle about grounding, disjunctive facts must be (partially) grounded in obtaining facts corresponding to their true disjuncts. In this case however, the fact about the essence of the variable explains the disjunctive fact directly without taking a detour through the true-disjunct-facts.

If Glazier is right, then there seems to be a problem with the two motivating arguments for the Linking Principle: If facts about essences do not generally ground the corresponding 'bare' facts ${ }^{31}$ about the relevant objects, then it might be argued that they also do not generally ground the truth of the propositions which state those facts. So, the objection goes, one cannot claim, as I did in Section 2.2. that facts about essence ground the truth of the propositions which describe the relevant essences.

To avoid this problem, one can adopt the view that facts about essences directly, rather than indirectly ground the truth of the propositions they contain. The idea is, again, that if a proposition $\langle p\rangle$ is true in virtue of the nature of $x x$, then there exists a fact that $\langle p\rangle$ is true in virtue of the nature of $x x$ which directly grounds the fact that $\langle p\rangle$ is true. Applied to Glazier's example, the idea is that the fact [It is essential to variable $v$ that $v$ has either value o or 1 ] directly grounds the (non-disjunctive!) fact [ $\langle v$ has either value o or 1$\rangle$ is true]. The disjunctive fact that the variable has either value o or 1 is in this case not needed as an intermediate step to (by transitivity) ground the truth of $\langle v$ has either value o or 1$\rangle$.

\section{An Application of the Linking Principle}

\subsection{Monotonicity of Essence}

In this section, I will argue that the Linking Principle together with two standard assumptions about grounding, namely that grounding is non-monotonic and that only facts which are relevant to a fact can be among its partial grounds, conflicts with the assumption that the constitutive Essentialist notion of 'true in virtue of the nature of' is monotonic. The argument for this claim itself turns out to be

31. In this context, 'bare fact' just means the following: If it is true in virtue of the nature of the $x x$ that $p$, then the bare fact corresponding to the fact about essence $[\langle p\rangle$ is true in virtue of the nature of $x x]$ is $[p]$. 
rather straight-forward, but as is often the case with philosophical arguments, the same cannot be said about the justification of its premises. Having already motivated and defended the Linking Principle against objections in the previous sections, my focus here will be on the monotonicity-assumption about 'true in virtue of the nature of':

Monotonicity of Essence For all objects $x x$, if $\langle p\rangle$ is true in virtue of the nature of $x x$, then for any objects $y y$, if all the $x x$ are among the $y y$, then $\langle p\rangle$ is true in virtue of the nature of $y y .^{32}$

The importance of this principles lies in the fact that it provides an answer to an important general question about plural essentiality, namely the question of whether the essences of single objects or of pluralities of objects are inherited by pluralities which contain them. Monotonicity of Essence gives a positive answer to this question. To illustrate this, let us assume that it is true in virtue of the nature of Socrates that he is human. If Monotonicity of Essence holds, then the same proposition is also true in virtue of the nature of any plurality of objects involving Socrates, such as that of him and Xanthippe.

What is the status of this principle? As a principle about consequential essence, it is uncontroversial. This is evident in Fine's logic of essence, the logic of the operator $\square_{x x}$, which is the formal counterpart of the consequential reading of 'true in virtue of the nature of'. Axiom II.v of the logical system developed in Fine (1995a) says that this operator is monotonic, that is, that if we have a plurality of objects $x x$ and another plurality of objects $y y$ which wholly contains the first, plus perhaps other objects, then if $\square_{x x} A$, then $\square_{y y} A .33$ This however does not settle our question, since, recall, the notion of essence involved in the Linking Principle and therefore the notion of essence with which we are concerned here is constitutive rather than consequential. These two notions are markedly different and one cannot in general infer the monotonicity of the one from the other. With this clarification made, I will from now on focus on the constitutive notion of Monotonicity of Essence. ${ }^{34}$

There is no standard view regarding whether the principle holds for constitutive essence. Since my aim in this section is to argue against it, I will for the

32. More formally stated using ' $x x \subseteq y y^{\prime}$ for 'all the $x x$ are among the $y y$ ': $\forall x x\left(\square_{x x} p \rightarrow\right.$ $\left.\forall y y\left(x x \subseteq y y \rightarrow \square_{y y} p\right)\right)$.

33. See Fine (1995a 247); note that Fine works with rigid predicates instead of pluralities, but this difference does not matter in the current context.

34. Note that Fine's original suggestion for an Essentialist definition of metaphysical necessity, which says that ' $[t]$ he metaphysically necessary truths can ... be identified with the propositions which are true in virtue of the nature of all objects whatever' (1994 9) arguably requires Monotonicity of Essence. See Correia 2012 640) and Michels (in press: Section 4). Fine works with a restricted consequential, rather than a constitutive notion of essence (see Fine 1995C: Section 3). 
rest of this subsection focus on considerations in favour of the principle, if only to illustrate why one might be inclined to accept it. 35

Are there philosophers who explicitly accept the principle? It might seem that Correia does, for the Essentialist theory of modality developed in Correia (2012) entails that the notion 'true in virtue of the nature of' is monotonic. The theory defines necessity in terms of an Essentialist notion which differs from 'true in virtue of the nature of', that of 'basic nature'. ${ }^{36}$ This notion is characterized in the following way: 'the basic nature of $x x$ [is] the plurality of propositions $\alpha$ such that for some $y y$ which is part of $x x, \alpha$ is basically essential to $y y^{\prime}$ (Correia 2012. 644 , notation adjusted). This characterization directly entails the monotonicity of this notion, or more precisely, of the notion of 'being part of the basic nature of'. Correia uses this notion together with a relativized notion of logical consequence to define Fine's notion 'true in virtue of the nature of'. Since both of these notions are monotonic, the defined version of Fine's notion also has this property. (See Correia 2012: 648.)

Does this mean that Correia accepts Monotonicity of Essence? Not quite, since neither the monotonic notion of basic nature, nor the defined monotonic version of 'true in virtue of the nature of', play the same role in Correia's version of the Essentialist theory of modality as 'true in virtue of the nature' does in Fine's version. The closest analogue to the latter is Correia's primitive Essentialist notion 'is basically essential to', so it would only be fair to call Correia a supporter of Monotonicity of Essence if that notion were monotonic. However, Correia (2012) remains silent about whether this notion has that property.

We should reflect on whether there is a general reason for Essentialists to accept this principle. Let me begin by considering the main intuitive reason for rejecting the principle: If it is true, there are lots of propositions which are true in virtue of the nature of a plurality of objects even though they only describe the essence of, for example, merely one of the objects involved, but not that of the whole plurality. An example would be 〈Socrates is human's being true in virtue of the nature of Socrates and the Eiffel Tower taken together, a proposition which partially captures Socrates's constitutive essence, but not that of the plurality of him and the Eiffel tower taken together. So, the thought goes, these propositions should not be part of this plural essence, since they fail to describe the essence of the whole plurality.

There is a prima facie plausible view of the essences of pluralities of objects which undermines this way of thinking. It is based on two ideas. The first is

35. When preparing the final version of this paper, I became aware of Zylstra (in press), which provides two arguments for Monotonicity of Essence. I will unfortunately not be able to discuss them here.

36. In Correia (in press), Correia instead uses the term 'constitutive essence' for the same primitive notion. 
that it is constitutively essential to pluralities of objects that those objects are among them which are actually among them. Accordingly, it is, for example, essential to Socrates and the Eiffel Tower together that Socrates and the Eiffel Tower are among them. The second idea is that it is furthermore part of the constitutive essence of a plurality of objects that these objects are the objects they are. According to this idea, the constitutively essential truths about the individual objects which are among a plurality also partly describe the constitutive essence of the whole plurality. On the resulting view, pluralities and the objects among them are essentially connected in an intimate way: The constitutive essential truths about the singular objects are a subset of the constitutive essential truths about the plurality or, to put it differently, the plurality inherits the constitutive essences of the objects among it. This view directly supports Monotonicity of Essence. ${ }^{37}$

\subsection{An Argument Against Monotonicity of Essence}

Essentialists who accept the view just described appear to have a good reason to accept Monotonicity of Essence. I will now argue that any Essentialist who accepts the Linking Principle should nonetheless reject this principle, since first, it conflicts with the assumption that a fact can only be grounded in facts which are relevant to it and second, it thereby undermines the main motivation for the standard assumption that grounding is non-monotonic. As a corollary, it also conflicts with the logically stronger assumption that grounding is minimal, which is endorsed by Audi. Here are these three further assumptions about grounding, spelled out more explicitly:

Relevance of Grounds For any plurality of facts $\mathrm{ff}$ and all facts $\mathrm{g}$, if $\mathrm{ff}$ fully ground $g$, then for all facts $h$ which are among the $f f, h$ is relevant to g. ${ }^{38}$

Non-Monotonicity of Grounding For some plurality of facts $\mathrm{ff}$ and some fact $g$, ff fully ground $g$ and there is some plurality of facts $h h$, such that all of the ff are among the hh, but hh do not ground g. ${ }^{39}$

37. Note that there is an alternative version of this view which does not directly support Monotonicity of Essence for constitutive essence. According to that view, even if it is constitutively essential to pluralities that the objects among them are among them and pluralities inherit the constitutive essences of these objects, the propositions which describe the constitutive essences of the singular objects are only consequentially, but not constitutively essential to the plurality comprising them. This version appears to be coherent and just as plausible as the version mentioned in the text. If one is drawn to a sparser view of plural constitutive essence, one may very well prefer it to the view which supports monotonicity. Thanks to Alexander Skiles and Tuomas Tahko for suggesting this view.

38. More formally: $\forall \mathrm{ff} \forall \mathrm{g}(\mathrm{ff} \rightarrow \mathrm{g} \rightarrow \forall \mathrm{h}(\mathrm{h} \sqsubseteq \mathrm{ff} \rightarrow \mathrm{hRg}))$

39. More formally: $\exists \mathrm{ff} \exists \mathrm{g}(\mathrm{ff} \vee \mathrm{g} \wedge \exists \mathrm{hh}(\mathrm{ff} \subseteq \mathrm{hh} \wedge \neg(\mathrm{hh} \triangleright \mathrm{g})))$.

Ergo $\cdot$ vol. 5, no. $30 \cdot 2018$ 
Minimality of Grounding For all pluralities of facts ff and all facts $g$, if ff fully ground $g$, then for any distinct plurality of facts hh including $\mathrm{ff}$, hh do not ground g. $4^{\circ}$

Relevance of Grounds captures the idea that any fact which contributes to (fully) grounding another fact has to contribute in some way to making it the case that the latter fact obtains. If one understands grounding to be a form of metaphysical explanation, then this requirement directly flows from a general requirement of explanatory relevance, which says that any element of the explanans of an explanation has to contribute in some way to explaining its explanandum (cf. Dasgupta 2014a: 4). Relevance of Grounds serves as the main motivation for Non-Monotonicity of Grounding, which, unlike Monotonicity of Essence, is part of the orthodoxy in the literature.$^{41}$ As Rosen puts it,

Intuitively, if $[p]$ is grounded in $\Gamma$, then every fact in $\Gamma$ plays some role in making it the case that $p$. Holding this fixed, monotonicity would entail that each fact plays a role in grounding every fact. And that is just not so. (2010. 116$)^{42}$

Fine even more explicitly appeals to the principle as a motivation for NonMonotonicity of Grounding when he states that the relationship of ground may not be preserved under the addition of grounds, since the new grounds may not be relevant to the truth of what is grounded' (2012b: 2). ${ }^{43}$ In other words, there is an intuitive reason to deny that grounding is monotonic, namely that the grounds have to be relevant to the grounded. This is exactly the idea captured by Relevance of Grounds.

Audi also appears to rely on Relevance of Grounds to motivate the stronger and more controversial principle Minimality of Grounding. ${ }^{44}$ According to Audi,

the fact that the shirt is both maroon and cotton does not ground the fact that it is red; the fact that it is cotton does no work with respect to making it red. So it appears that a ground of some fact must be minimal in the sense of containing only elements that jointly suffice to bring it about that the fact in question obtains. 2012b; 699)

If we understand 'does no work with respect to making it' in the manner suggested by Dasgupta and Rosen, Audi's motivating argument for Minimality of

40. More formally: $\forall \mathrm{ff} \forall \mathrm{g}(\mathrm{ff} \rightarrow \mathrm{g} \rightarrow \forall \mathrm{hh}(\mathrm{ff} \subsetneq \mathrm{hh} \rightarrow \neg(\mathrm{hh} \triangleright \mathrm{g}))$ ). (See Audi 2012b 699.)

41. See however Raven (2013 198-199) for a critical discussion.

42. See also Fine (2012a 56).

43. Note that despite the formulation, Fine is not referring to truth-grounds in the last part of the quoted passage, but simply to grounds for the relevant fact.

44. Dixon 2015 Section 5) and Fine 2012a 57) argue against Minimality of Grounding. 
Grounding is another application of Relevance of Grounds. This is noteworthy, since Minimality is a logically stronger principle than Non-Monotonicty of Grounding, in the sense that it implies, but is not implied by the latter. To further bring out the contrast between the two principles, if we presuppose Relevance of Grounds, the difference between accepting the former and accepting the latter is that of allowing instances of grounding which are preserved under the addition of relevant grounds versus denying that there are such instances, because nothing short of the maximal plurality of relevant grounds is good enough to give us an instance of grounding in the first place.

Now on to the main argument of this section. It proceeds in two steps, one of which takes the form of a reductio argument. ${ }^{45}$ The reductio assumes the Linking Principle and Monotonicity of Essence and derives a counterexample to Relevance of Grounds, thereby undermining the motivation for Non-Monotonicity and Minimality of Grounding: Suppose that it is true in virtue of the nature of the number two that it is even. By Monotonicity of Essence, that the number two is even is also true in virtue of the nature of the number two and the Tower Bridge taken together. By the Linking Principle, there are facts involving the number two and the Tower Bridge which together ground the truth of / The number two is even $\rangle$. By Relevance of Grounds, both of these facts are relevant to the latter fact. However, no fact about the Tower Bridge is relevant to or, to put it differently, plays a role in making it the case that 〈The number two is even〉 is true. Contradiction. ${ }^{46}$

This reductio-argument shows that the Linking Principle and Monotonicity of Essence together conflict with Relevance of Grounds and thereby with the main (and seemingly only) motivation for both Non-Monotonicity and Minimality of Grounding. Based on the arguments in favour of and responses to objections to the Linking Principle presented in the previous sections, I will here assume, and this is the second step of my argument, that it is in better standing than Monotonicity of Essence. Since Relevance of Grounds, as well as Non-Monotonicity and, to a lesser degree, since only Audi explicitly accepts it, Minimality of Ground, are well-established assumptions about grounding, the final conclusion which I want to draw from the reductio-argument is that Monotonicity of Essence has to be rejected.

45. Thanks to Benjamin Schnieder for suggesting an argument along the following lines.

46. One might wonder whether a parallel argument against the monotonicity of 'true in virtue of the nature of' could be run based on a relevance principle which directly applies to this notion. The problem with this idea is that the sort of relevance-principle which we could formulate based on the standard views about what is essential to what (derived from Fine's 1994 ideas about essential connections between objects and Fine's 1995 b view of the relation between ontological dependence and essence) does not settle whether the notion is monotonic. See also the introductory paragraphs of Zylstra (in press). 


\section{Truth-Grounding, Grounding, and Truthmaking}

There is a way to resist my reading of the reductio-argument, namely by denying the idea that truth-grounding is grounding, or, for short, truth-grounding as grounding. The main argument against truth-grounding as grounding is a conditional argument due to Tahko (2013).

Tahko argues that if grounding is univocal, that is, if there is only one variety of grounding, then one has to deny that truth-grounding is grounding or that grounding is transitive, or both. The disjunctive consequent of this conditional is supported by an argument against the transitivity of truth-grounding. This argument is based on the assumption that truth-grounding can be used to explicate truthmaking, which Tahko accepts for the sake of his argument.

The argument runs as follows: Consider the proposition $\langle b$ exists $\rangle$, where $b$ names a bottle of beer. We may assume that the fact that this proposition is true is grounded in [ $b$ exists]. This fact in turn is partially grounded in the fact that $b$ has a stable macrophysical structure. The latter fact is furthermore partially grounded in the fact that the Pauli Exclusion Principle (an important physical principle regarding the stability of matter) holds. By the transitivity of grounding, the fact that the Pauli Exclusion Principle holds (partially) grounds [ $\langle b$ exists $\rangle$ is true]. Tahko then argues that this cannot be the case, since this grounding claim does not satisfy a strict relevance requirement for truth-grounding which says that if $[q]$ is not strictly relevant to the truth of $\langle p\rangle$, then it is not the case that $[q]$ helps ground $[\langle p\rangle$ is true].

To understand the last move in the argument, we have to keep in mind that it is premised on the idea that truth-grounding explicates truth-making. The strict relevance requirement ensures that the truth-grounding-based explication accounts for a task which truth-making is supposed to fulfil: 'A central task of truthmaking theory is to provide an explanation as to what makes one rather than another proposition true, call it the discernment task of truthmaking theory' (Tahko 2013 337). Given this requirement, the fact that the Pauli Exclusion Principle holds cannot be a truth-ground for $\langle b$ exists $\rangle$, since it does not discern between the truth of $\langle b$ exists $\rangle$ and a proposition about a distinct bottle of beer $c,\langle c$ exists $\rangle$. This, argues Tahko, leaves us two options: We can prioritize staying in line with standard truthmaker theory regarding the discernment task and reject the transitivity of grounding, or we can stay in line with standard grounding-theory which endorses transitivity and reject truth-grounding as grounding.

The objection to my argument is based on the second option. If truthgrounding is not grounding, the counterexample to Relevance of Grounds can be rejected for the simple reason that it does not involve grounding. A further and even more worrying implication of the objection is that it questions the general relevance of the Linking Principle to grounding. 
Fortunately, there is a way around the dilemma posed by Tahko's argument. ${ }^{47}$ The grounding-chain on which Tahko's example is based involves an instance of partial grounding between the fact that the Pauli Exclusion Principle holds and [ $b$ exists] and an instance of full grounding between [ $b$ exists] and [ $\langle b$ exists $\rangle$ is true]. The instance of truth-grounding Tahko relies on to support his conclusion is derived from this chain by transisitivity. This derived instance is an instance of partial grounding. ${ }^{4}$ Extrapolating from Tahko's principle (TG), which says that if [ $a$ exists] partially grounds [ $\langle p\rangle$ is true], then [ $a$ exists] is a (partial) truth-ground for $\langle p\rangle$ (see Tahko 2013: 333, notation adjusted), we can infer that the fact that the Pauli Exclusion Principle holds is a partial truth-ground for $\langle b$ exists $\rangle$, that is, that it helps to make the proposition true. Helping to make true is of course not the same as making true. That the fact does not qualify as a truthmaker for the proposition (i.e., as a full truth-ground for it) on its own is clear, since the Pauli Exclusion Principle alone does not tell us anything about whether it is true that a particular macroscopic object exists. This means that one can resist Tahko's argument by insisting that the strict relevance requirement, that is, the requirement to fulfil the discernment task, applies only to full truth-grounds, but not to partial truth-grounds.

The resulting weaker version of the strict relevance requirement is independently plausible, as examples such as the following illustrate: For any composite object, we may assume that each fact about the exact location of one of its proper parts serves as a partial truth-ground for a proposition stating the exact location of the composite object. Only all such facts taken together can serve as the proposition's 'full' truth-ground and thereby provide one of its truthmakers. None of the particular facts alone will fulfil the discernment task, since it might as well serve as a partial truth-ground for a proposition which states the exact location of a distinct composite object which has the relevant object as a part. The same

47. I owe the following response to an anonymous referee. Before adopting this response, I argued instead that one can avoid Tahko's argument by denying that truth-grounding explicates truthmaking. I take the following to be a better response to Tahko, since it allows one to remain neutral regarding this explication-claim. In this context it should be noted that there are arguments against the similar idea that truthmaking can be defined in terms of grounding. Audi (2012a 113) for example argues that instances of truthmaking involve a definitional connection between an entity and a truth and not the sort of explanatory connection which he assumes to be characteristic of instances of grounding. Griffith 2014 Section II) critically discusses a range of different grounding-based definitions of truthmaking and in particular raises three objections to a definition which is based on the view that grounding is a relation between facts, i.e., the view which I have been working with in this paper. I will not engage with these arguments here.

48. This follows from a general principle about grounding which, e.g., holds in Fine's pure logic of ground. (See, e.g., Fine 2012a Section 1.6.) To see why we generally do not get an instance of full grounding from an instance of partial grounding plus an instance of full grounding by transitivity, consider the following counterexample: [ $p$ ] partially grounds $[p \wedge q]$, which fully grounds $[(p \wedge q) \vee r]$, but $[p]$ only partially, but not fully grounds $[(p \wedge q) \vee r]$. 
point can be made in a more general fashion: It is plausible to assume that in cases in which the discernment task is fulfilled, the truth has to be necessitated by its truthmaker, ${ }^{49}$ but such a necessary connection need not obtain if a fact merely helps ground or make a proposition true, but does not do so on its own. The threat to the argument of the previous section and more generally to the Linking Principle posed by Tahko's argument can hence be defused without giving up on the idea that truthmakers have to be able to fulfil the discernment task.

\section{Conclusion}

The Linking Principle tells us that the truth of any proposition which expresses an essential truth about some objects is fully grounded in some facts involving these objects. In this paper, I have first motivated and then defended the Linking Principle against objections. Relying on the standard assumption that grounds have to be relevant to what they ground, I have then used the principle to argue that the Essentialist notion 'true in virtue of the nature of' is non-monotonic. Finally, I have argued that the principle is not threatened by Tahko's (2013) conditional argument against the idea that truth-grounding is grounding. This all suggests that the Linking Principle captures an important connection between essence, truth, and grounding which has substantial implications for all three notions.

\section{Acknowledgments}

Thanks to the people who discussed a version of this paper at the eidos seminar, to Philipp Blum, Claudio Calosi, Fabrice Correia, Ghislain Guigon, Kevin Mulligan, Paolo Natali, Benjamin Neeser, Jan Plate, and to an audience at the Truth and Grounds conference in Ascona where I presented a very different earlier version of the paper, in particular Lorenzo Casini, Catherine Diehl, Kit Fine, Michael Raven, Julio de Rizzo, Benjamin Schnieder, Jonas Werner, and Nathan Wildman. I am furthermore grateful to several anonymous referees who reviewed the paper for this and other journals and to Tuomas Tahko for written comments. Finally, I thank Alexander Skiles for both commenting on earlier versions of the paper and for several very helpful discussions. I gratefully acknowledge financial support of the Swiss National Science Foundation (Project 'Indeterminacy and Formal Concepts', Grant-No. 156554, University of Geneva, principal investigator: Kevin Mulligan).

49. In the sense that necessarily, if the truthmaker exists, the relevant proposition is true. 


\section{References}

Armstrong, David M. (2004). Truth and Truthmakers. Cambridge University Press. Audi, Paul (2012a). A Clarification and Defense of the Notion of Grounding. In Fabrice Correia and Benjamin Schnieder (Eds.), Metaphysical Grounding: Understanding the Structure of Reality (101-121). Cambridge University Press.

Audi, Paul (2012b). Grounding: Toward a Theory of the In-Virtue-Of Relation. Journal of Philosophy, 109(12), 685-711.

Barrio, Eduardo and Gonzalo Rodríguez-Pereyra (2015). Truthmaker Maximalism Defended Again. Analysis, 75(1), 3-8.

Beddor, Bob (2015). Evidentialism, Circularity, and Grounding. Philosophical Studies, 172(7), 1847-1868.

Bennett, Daniel (1969). Essential Properties. Journal of Philosophy, 66(15), 487-499. Bliss, Ricki and Kelly Trogdon (2016). Metaphysical Grounding. In Edward N. Zalta (Ed.), The Stanford Encyclopedia of Philosophy (Winter 2016 ed.). Retrieved from https://plato.stanford.edu/archives/win2016/entries/grounding/

Cameron, Ross P. (2008). Truthmakers and Modality. Synthese, 164(2), 261-280.

Carnap, Rudolf (1932). Überwindung der Metaphysik durch logische Analyse der Sprache. Erkenntnis, 2(1), 219-241.

Carnino, Pablo (2015). On the Reduction of Grounding to Essence. Studia Philosophica Estonica, 7(2), 56-71.

Chisholm, Roderick M. (1973). Parts as Essential to Their Wholes. The Review of Metaphysics, 26(4), 581-603.

Clark, Michael J. and David Liggins (2012). Recent Work on Grounding. Analysis, $72(4), 812-823$.

Contessa, Gabriele (2010). Modal Truthmakers and Two Varieties of Actualism. Synthese, 174(3), 341-353.

Correia, Fabrice (2000). Propositional Logic of Essence. Journal of Philosophical Logic, 29(3), 295-313.

Correia, Fabrice (2006). Generic Essence, Objectual Essence, and Modality. Noûs, $40(4), 753-767$.

Correia, Fabrice (2012). On the Reduction of Necessity to Essence. Philosophy and Phenomenological Research, 84(3), 639-653.

Correia, Fabrice (2013). Metaphysical Ground and Essence. In Miguel Hoeltje, Benjamin Schnieder, and Alexander Steinberg (Eds.), Varieties of Dependence. Ontological Dependence, Grounding, Supervenience, Response-Dependence (271296). Philosophia.

Correia, Fabrice (in press). More on the Reduction of Necessity to Essence. In Mircea Dumitru (Ed.), Metaphysics, Meaning, and Modality. Themes from Kit Fine. Oxford University Press.

Correia, Fabrice and Benjamin Schnieder (2012a). Grounding: An Opinionated 
Introduction. In Fabrice Correia and Benjamin Schnieder (Eds.), Metaphysical Grounding: Understanding the Structure of Reality (1-36). Cambridge University Press.

Correia, Fabrice and Benjamin Schnieder (2012b). Metaphysical Grounding: Understanding the Structure of Reality. Cambridge University Press.

Correia, Fabrice and Alexander Skiles (in press). Grounding, Essence, and Identity. Philosophy and Phenomenological Research.

Cowling, Sam (2013). The Modal View of Essence. Canadian Journal of Philosophy, $43(2), 248-266$.

Dasgupta, Shamik (2014a). On the Plurality of Grounds. Philosophers' Imprint, 14(20). Retrieved from http://hdl.handle.net/2027/spo.3521354.0014.020

Dasgupta, Shamik (2014b). The Possibility of Physicalism. Journal of Philosophy, $111(9 / 10), 557-592$.

Dasgupta, Shamik (2016). Metaphysical Rationalism. Nô̂s, 50(2), 379-418.

Della Rocca, Michael (2014). Razing Structures to the Ground. Analytic Philosophy, 55(3), 276-294.

Dixon, T. Scott (2015). Grounding and Supplementation. Erkenntnis, 81(2), 375389.

Ellis, Brian (2001). Scientific Essentialism. Cambridge University Press.

Fine, Kit (1994). Essence and Modality. Philosophical Perspectives, 8(Logic and Language), 1-16.

Fine, Kit (1995a). The Logic of Essence. Journal of Philosophical Logic, 24(3), $241-273$.

Fine, Kit (1995b). Ontological Dependence. Proceedings of the Aristotelian Society, 95, 269-290.

Fine, Kit (1995c). Senses of Essence. In Walter Sinnott-Armstrong (Ed.), Modality, Morality, and Belief: Essays in Honor of Ruth Barcan Marcus (53-73). Cambridge University Press.

Fine, Kit (2000). Semantics for the Logic of Essence. Journal of Philosophical Logic, 29(6), 543-584.

Fine, Kit (2012a). Guide to Ground. In Fabrice Correia and Benjamin Schnieder (Eds.), Metaphysical Grounding: Understanding the Structure of Reality (37-80). Cambridge University Press.

Fine, Kit (2012b). The Pure Logic of Ground. The Review of Symbolic Logic, 5(1), $1-25$.

Fine, Kit (2015). Unified Foundations for Essence and Ground. Journal of the American Philosophical Association, 1(2), 296-311.

Garcia-Carpintero, Manuel (2007). A Non-Modal Conception of Secondary Properties. Philosophical Papers, 36(1), 1-33.

Glazier, Martin (2016). Essentialist Explanation. Philosophical Studies, 174(11), 2871-2889. 
Gorman, Michael (2005). The Essential and the Accidental. Ratio, 18(3), 276-289. Griffith, Aaron M. (2014). Truthmaking and Grounding. Inquiry, 57(2), 196-215. Koslicki, Kathrin (2012). Varieties of Ontological Dependence. In Fabrice Correia and Benjamin Schnieder (Eds.), Metaphysical Grounding: Understanding the Structure of Reality (186-213). Cambridge University Press.

Kroedel, Thomas and Moritz Schulz (2016). Grounding Mental Causation. Synthese, 193(6), 1909-1923.

Linnebo, Øystein. (2017). Plural Quantification. In Edward N. Zalta (Ed.), The Stanford Encyclopedia of Philosophy (Summer 2017 ed.). Retrieved from https: //plato.stanford.edu/archives/sum2017/entries/plural-quant/

Lowe, E. J. (2008). Two Notions of Being: Entity and Essence. Royal Institute of Philosophy Supplements, 83(62), 23-48.

MacBride, Fraser (2013). Truthmakers. In Edward N. Zalta (Ed.), The Stanford Encyclopedia of Philosophy (Fall 2016 ed.). Retrieved from https://plato.stanford. edu/archives/fall2016/entries/truthmakers /

Mackie, Penelope (1994). Sortal Concepts and Essential Properties. Philosophical Quarterly, 44(176), 311-333.

Mellor, D. Hugh (2003). Real Metaphysics: Replies. In Hallvard Lillehammer and Gonzalo Rodríguez-Pereyra (Eds.), Real Metaphysics: Essays in Honour of D. H. Mellor (212-38). Routledge.

Michels, Robert (in press). On How (Not) to Define Modality in Terms of Essence. Philosophical Studies.

Milne, Peter (2005). Not Every Truth Has a Truthmaker. Analysis, 65(3), 221-224. Mulligan, Kevin, Peter Simons, and Barry Smith (1984). Truth-makers. Philosophy and Phenomenological Research, 44(3), 187-321.

Oderberg, David S. (2011). Essence and Properties. Erkenntnis, 75(1), 85-111. Oliver, Alex and Timothy Smiley (2013). Plural Logic. Oxford University Press.

Quine, Willard V. O. (1948). On What There Is. In Willard V. O. Quine (Ed.), From a Logical Point of View (2nd ed., 1-19). Harvard University Press.

Raven, Michael J. (2013). Is Ground a Strict Partial Order? American Philosophical Quarterly, 50(2), 191-199.

Raven, Michael J. (2015). Ground. Philosophy Compass, 10(5), 322-333.

Robertson, Teresa and Philip Atkins (2013). Essential vs. Accidental Properties. In Edward N. Zalta (Ed.), The Stanford Encyclopedia of Philosophy (Spring 2018 ed.). Retrieved from https://plato.stanford.edu/archives/spr2018/entries/ essential-accidental/

Roca-Royes, Sonia (2011). Essential Properties and Individual Essences. Philosophy Compass, 6(1), 65-77.

Rodríguez-Pereyra, Gonzalo (2002). Resemblance Nominalism: A Solution to the Problem of Universals. Oxford University Press.

Rodríguez-Pereyra, Gonzalo (2006a). Truthmaker Maximalism Defended. Analysis, 
66(3), 260-264.

Rodríguez-Pereyra, Gonzalo (2006b). Truthmakers. Philosophical Compass, 1(2), 186-200.

Rosen, Gideon (2010). Metaphysical Dependence: Grounding and Reduction. In Bob Hale and Aviv Hoffmann (Eds.), Modality: Metaphysics, Logic, and Epistemology (109-135). Oxford University Press.

Schaffer, Jonathan (2009). On What Grounds What. In David Manley and David J. Chalmers and Ryan Wasserman (Eds.), Metametaphysics: New Essays on the Foundations of Ontology (347-383). Oxford University Press.

Schnieder, Benjamin (in press). Grounding and Dependence. Synthese.

Skiles, Alexander (2015). Essence in Abundance. Canadian Journal of Philosophy, 45(1), 100-122.

Tahko, Tuomas E. (2013). Truth-Grounding and Transitivity. Thought, 2(4), 332340.

Trogdon, Kelly (2013a). Grounding: Necessary or Contingent? Pacific Philosophical Quarterly, 94(4), 465-485.

Trogdon, Kelly (2013b). An Introduction to Grounding. In Miguel Hoeltje, Benjamin Schnieder, and Alexander Steinberg (Eds.), Varieties of Dependence: Ontological Dependence, Grounding, Supervenience, Response-Dependence (97-122). Philosophia.

Väyrynen, Pekka (2013). Grounding and Normative Explanation. Proceedings of the Aristotelian Society, Supplementary Volume, 87(1), 155-178.

Wildman, Nathan (2013). Modality, Sparsity, and Essence. Philosophical Quarterly, $63(253), 760-782$.

Wilson, Jessica M. (2014). No Work for a Theory of Grounding. Inquiry, 57(5-6), $535-579$.

Wilson, Jessica (2017). Determinables and Determinates. In Edward N. Zalta (Ed.), The Stanford Encyclopedia of Philosophy (Spring 2017 ed.). Retrieved from https://plato.stanford.edu/archives/spr2017/entries/ determinate-determinables/

Zylstra, Justin (in press). Collective Essence and Monotonicity. Erkenntnis. 